\title{
Optical Coherence Tomography: a non-invasive technique applied to conservation of paintings
}

\author{
Haida Liang ${ }^{* a}$, Marta Gomez Cid ${ }^{\mathrm{b}, \mathrm{c}}$, Radu Cucu $^{\mathrm{b}}$, George Dobre $^{\mathrm{b}}$, Boris Kudimov ${ }^{\mathrm{b}}$, Justin Pedro ${ }^{\mathrm{d}}$, \\ David Saunders ${ }^{\mathrm{e}}$, John Cupitt ${ }^{\mathrm{e}}$, Adrian Podoleanu ${ }^{\mathrm{b}}$ \\ ${ }^{a}$ School of Biomedical and Natural Sciences, Nottingham Trent University, Clifton Lane, \\ Nottingham NG11 8NS, UK \\ ${ }^{\mathrm{b}}$ School of Physical Sciences, University of Kent, Canterbury, CT2 7NR, UK \\ ${ }^{c}$ Facultade de Ciencias de Ourense, Universidade de Vigo, As Lagoas s/n, 32004, Ourense, Spain \\ ${ }^{\mathrm{d}}$ Ophthalmic Technologies Inc, Toronto, Canada \\ ${ }^{\mathrm{e}}$ Scientific Department, The National Gallery, London WC2N 5DN, UK
}

\begin{abstract}
It is current practice to take tiny samples from a painting to mount and examine in cross-section under a microscope. However, since conservation practice and ethics limit sampling to a minimum and to areas along cracks and edges of paintings, which are often unrepresentative of the whole painting, results from such analyses cannot be taken as representative of a painting as a whole. Recently in a preliminary study, we have demonstrated that near-infrared Optical Coherence Tomography (OCT) can be used directly on paintings to examine the cross-section of paint and varnish layers without contact and the need to take samples. OCT is an optical interferometric technique developed for in vivo imaging of the eye and biological tissues; it is essentially a scanning Michelson's interferometer with a 'broadband' source that has the spatial coherence of a laser. The low temporal coherence and high spatial concentration of the source are the keys to high depth resolution and high sensitivity $3 \mathrm{D}$ imaging. The technique is non-invasive and noncontact with a typical working distance of $2 \mathrm{~cm}$. This non-invasive technique enables cross-sections to be examined anywhere on a painting. In this paper, we will report new results on applying near-infrared en-face OCT to paintings conservation and extend the application to the examination of underdrawings, drying processes, and quantitative measurements of optical properties of paint and varnish layers.
\end{abstract}

Keywords: optical coherence tomography, low coherence interferometry, art conservation, wetting, drying, paint, varnish, infrared imaging, infrared reflectography, 3D imaging, refractive index, confocal microscopy

\section{INTRODUCTION}

Scientific examination of easel paintings is carried out routinely in major galleries and museums to assist in conservation treatment and as part of technical or art historical examinations. Increasingly emphasis is being placed on non-destructive and non-invasive methods of analysis and preventive conservation. However, to study the paint and varnish layers, it is still current practice to take tiny samples from a painting to mount and examine in cross-section under a microscope. Since the 1930s, the examination of cross-sections has been essential to identifying the pigments and media, their method of application, including relative thickness, the composition of superimposed layers and signs of deterioration and previous alteration ${ }^{1}$. However, since conservation practice and ethics limit sampling to a minimum (normally less than one square millimetre in size) and to areas along cracks and edges of paintings, which are often unrepresentative of the whole painting, results from such analyses cannot be taken as representative of the painting as a whole. A non-invasive technique for examining the paint and varnish layers would not only reduce the need to take samples from paintings, but also enable examinations on any area of a painting.

Currently, routine non-invasive methods of examination include X-radiography, infrared reflectography, macrophotography, UV-fluorescence and raking light imaging. The last three methods give information mostly on the conditions of the surface of a painting. X-radiography is routinely employed to examine the structure of the support of a

*haida.liang@ntu.ac.uk; phone 44115848 3448; fax 44115848 6636; also visiting lecturer at the University of Kent.

Optical Methods for Arts and Archaeology, edited by Renzo Salimbeni, Luca Pezzati, Proceedings of SPIE Vol. 5857

(SPIE, Bellingham, WA, 2005) · 0277-786X/05/\$15 · doi: 10.1117/12.612591 
painting, as well as details of areas painted with pigments containing heavy elements. Infrared reflectography is one of the most useful techniques for art historians studying the preparatory drawings or underdrawings underneath the painted layers, which would be otherwise invisible to the eye. Both X-radiography and infrared reflectography reduce the 3D information of a painting into $2 \mathrm{D}$, thus losing the detailed information perpendicular to the painting plane.

Laser interferometry such as holography and speckle interferometry have been applied to works of art to detect structural defects ${ }^{2,3}$. However, since these methods employ a laser source that has high temporal coherence, they do not offer the ability to distinguish between successive reflective layers.

In the context of an EU-funded project, CRISATEL, we have conducted a pilot study with the leading ellipsometer company SOPRA in Paris to evaluate whether ellipsometry can be used to measure varnish thickness and optical properties on paintings. It was concluded that while it is possible to measure thin smooth varnish layers on a smooth glass substrate or a smooth machine painted paint layer ${ }^{4}$, varnish layers on real paintings are too thick and too rough to be measured by any ellipsometer (including those operating in the infrared). Ellipsometry is mainly designed for the semi-conductor industry, to measure ultra-smooth and relatively thin transparent film deposits such as $\mathrm{SiO}_{2}$ on metal substrates 5 .

Confocal microscopy has been tried on tiny samples for depth resolved images. Theoretically it is a non-destructive and non-invasive technique, but in practice hazardous because of the close working distance (a few mm) required for high depth resolution imaging ${ }^{6}$.

Recently a novel technique - Optical Coherence Tomography (OCT) has been successfully applied to art conservation to produce cross-sectional images of paintings and archaeological objects non-invasively ${ }^{7,8,9}$. OCT is basically a fast high-resolution 3-D scanning Michelson's interferometer ${ }^{10}$. It was developed specifically for producing cubes of 3-D images for examining the eye and other biological tissues. Optical Coherence Tomography (OCT) uses the low coherence interferometry principle, where the depth resolution is not like in confocal microscopy given by the numerical aperture of the microscope objective, but by the width of the source spectrum. The coherence properties play an essential role - to achieve high depth resolution short coherence length or wide-band sources are required. Wide-band sources such as superluminescent diode (SLD), Kerr lens mode-locked laser and supercontinuum sources have been used for this purpose. Sub-micron resolutions are achievable using specialized ultra-wide band sources ${ }^{11}$. OCT gives higher dynamic range images than confocal microscopy - a reflectivity below $10^{-13}$ could in principle be measured. It is recognized that in comparison with confocal microscopy, OCT gives double the penetration depth in highly scattering samples such as paint layers since it takes advantage of the coherence properties of light and registers only coherent signals. Since OCTs are designed for in vivo examinations of a highly sensitive organ - the eye, care must be taken to avoid any potential risk of contact. Hence it has the added advantage of a comfortably remote working distance, typically $\sim 2 \mathrm{~cm}$.

\section{THE INSTRUMENTS}

In this work we have used a number of different OCT systems that were assembled by the Applied Optics Group of the University of Kent. The optical configuration is similar to that presented in ${ }^{12}$, using two single mode directional couplers with a superluminiscent diode as the source. Unlike a conventional time-domain OCT ${ }^{13}$ where a stack of cross-section images are taken one after another to create a 3-D image, an en-face OCT takes images in planes parallel to the painting surface one after another in depth ${ }^{14}$ which is particularly convenient for the examination of paintings. This is similar to the procedure used in any confocal microscope, where the fast scanning is en-face and the depth scanning (focus change rather than a change in the reference arm length in the case of an OCT) is much slower (at the frame rate $)^{15}$. The en-face scans provide an instant comparison to the familiar sight of a painting. Features seen with the naked eye could easily be compared with features hidden in depth. Sequential and rapid switching between the en-face regime and the cross-section regime, specific for the en-face OCT systems ${ }^{16}$ developed by us, represents a significant advantage in the non-invasive imaging of paintings. Images with different orientations can be obtained using the same system with no need to move the painting. 
As shown in Fig.1, in the en-face regime, the frame grabber is controlled by signals from the generators driving the $\mathrm{X}$ scanner and the Y-scanner. One galvo is driven with a ramp at $500 \mathrm{~Hz}$ and the other galvo-scanner with a ramp at $2 \mathrm{~Hz}$. In this way, an en-face image, in the plane $(\mathrm{x}, \mathrm{y})$ is generated at constant depth. The next en-face image at a new depth is then generated by moving the translation stage in the reference arm of the interferometer and repeating the (x,y) scan. Ideally, the depth interval between successive frames should be much smaller than the system resolution in depth and the depth change applied only after the entire en-face image has been collected. However, in practice, to speed up the acquisition, the translation stage was moved continuously. In the images presented below, no other phase modulation was employed apart from that introduced by the X-galvanometer scanner. In Podoleanu et al. ${ }^{12}$, we demonstrated the role played by the image size in balancing the effects of an external phase modulator and of the modulation produced by the transversal scanner. If the image is sufficient large, then the distortions introduced by not using a phase modulator are insignificant.

In the cross-section regime, the frame grabber is controlled by signals from the generator driving the $\mathrm{X}$-scanner (or the Y-scanner) with a ramp at $500 \mathrm{~Hz}$ and the translation stage moving over the depth range required in $0.5 \mathrm{~s}$. In this case, an OCT cross-section image is produced either in the plane $(\mathrm{x}, \mathrm{z})$ or $(\mathrm{y}, \mathrm{z})$.

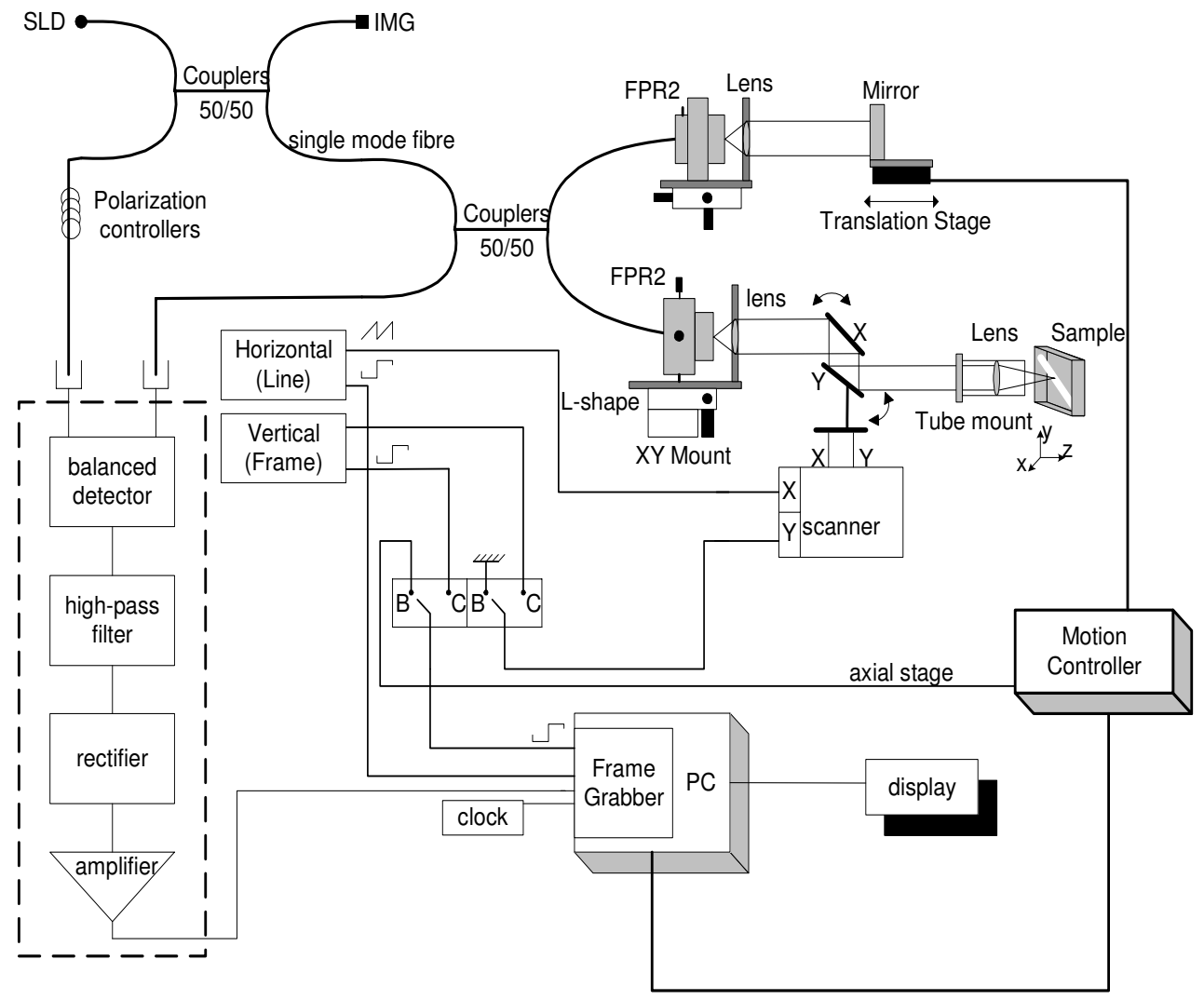

SLD = superluminescent diode

$\mathrm{IMG}=$ index matching gel

Fig. 1. Optical coherence tomography system architecture for the examination of paintings

The three en-face OCT systems used in this research are listed below in Table 1 where the transverse resolution (i.e. in the en-face plane) and the depth resolution in air are given along with the wavelength of the SLD sources. System 1 has a low numerical aperture (NA) interface optics which gives a large field of view of $1 \mathrm{~cm}$ by $1 \mathrm{~cm}$. System 2 has a medium NA interface optics which gives a better transverse resolution. System 3 uses dual SLD sources at $1300 \mathrm{~nm}$ to 
increase the total bandwidth giving a better depth resolution than System 1. System 3 has a high NA interface optics giving the best transverse resolution. Systems 1 and 2 have typical working distances of 2 to $3 \mathrm{~cm}$ and in the case of System 3 it is less than $1 \mathrm{~cm}$. The transversal resolution was evaluated by scanning a standard resolution target at the focal plane of the interface lens. With the transversal scanning switched off, the depth resolution was measured by placing a plane mirror in the object arm and recording the correlated signal strength as a function of the reference arm length. In order to image different objects, adjustable interface optics was designed.

Table 1 Specifications of the OCT systems

\begin{tabular}{cccc}
\hline OCT system & wavelength $(\mathrm{nm})$ & depth resolution $(\mu \mathrm{m})$ & transverse resolution $(\mu \mathrm{m})$ \\
\hline 1 & 1300 & 20 & 25 \\
2 & 850 & 18 & 15 \\
3 & 1300 & 15 & 5 \\
\hline
\end{tabular}

\section{RESULTS}

\subsection{Non-invasive imaging of cross-sections}

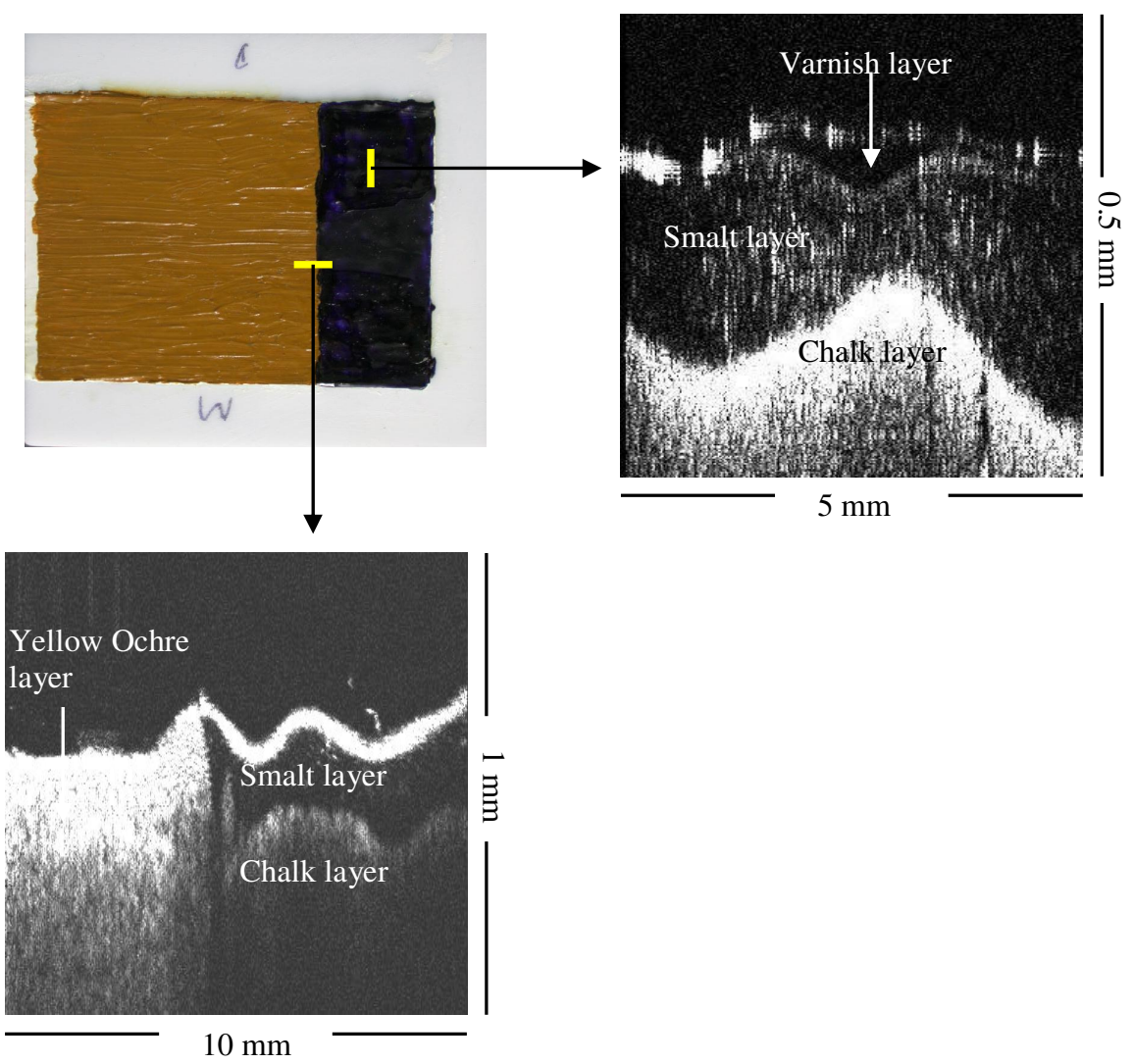

Fig. 2 Top-Left: A hand painted test panel; Top-Right: An OCT cross-section image obtained with System 2; Bottom-Left: An OCT cross-section image obtained with System 1. 
A test panel was prepare with a traditional ground layer of chalk mixed with rabbit skin glue, on top of which a layer of yellow ochre in linseed oil was painted on the left and smalt in linseed oil was painted on the right. The top third of the panel was varnished with a thin layer of dammar varnish, the middle part of the panel is left unvarnished and the bottom third of the panel was varnished with mastic. Both mastic and dammar varnish are traditional varnishes for paintings. A cross-section image of a scan in the varnished smalt part of the panel was obtained with System 2 showing the transparent varnish layer, the semi-transparent smalt layer and the chalk ground layer (see Fig. 2 Top-Right). A crosssection image with slightly lower resolution was obtained with System 1 for the yellow ochre/smalt boundary on the unvarnished part of the panel (Fig. 2 Bottom-Left), which shows yellow ochre to be a highly scattering medium compared with smalt.

Cross-sectional images of an unaccessioned $18^{\text {th }}$ century painting are shown in Fig. 3. The upper scan reveals that part of the varnish has been detached from the painting. The thickness of the varnish near the edge of the detachment as well as its refractive index can be estimated using the method described in the next section. The lower scan shows that varnish was applied after the damage which resulted in paint loss; it was applied on the bare canvass on the lower part of the scan but on top of the paint on the upper region of the scan.
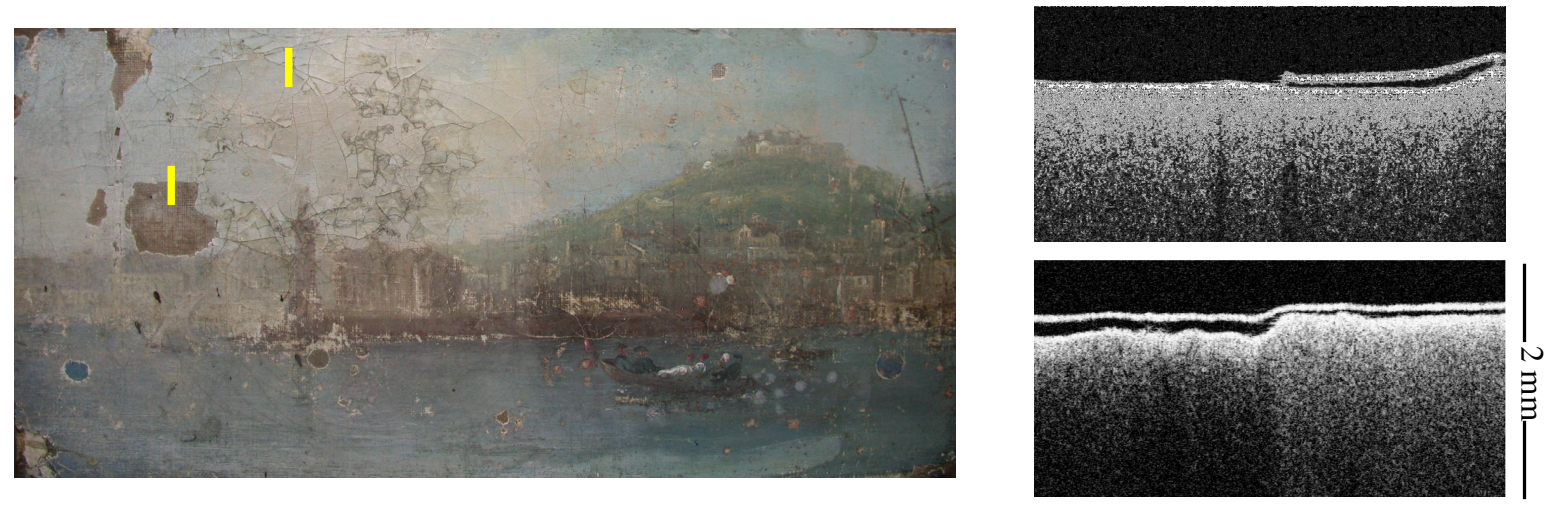

$10 \mathrm{~mm}$

Fig. 3 Left: An $18^{\text {th }}$ century panel painting; Top-Right: A cross-section image of a scan along the top line-segment marked on the painting; Bottom-Right: A cross-section image of along the lower line-segment on the painting. Both images were obtained with System 1 .

\subsection{Imaging of underdrawings}

Fig. 4 shows an example of a series of images taken in depth of a small region of a painted board. A stack of 80 en-face images were collected while moving the reference stage at $50 \mu \mathrm{m} / \mathrm{s}$ which means $25 \mu \mathrm{m}$ in depth between the frames for a frame rate of $2 \mathrm{~Hz}$. As images are taken deeper into the painting, underdrawings (preparatory drawings before the application of paint) are revealed. Fig. 5 shows that the dynamic range and resolution of the OCT images of underdrawings surpass any conventional infrared images. The high dynamic range is because interferometers register only coherent signals; hence only back-scattered light from the layer that matches (within the coherence length) the reference path length is registered. Back-scattered light from the other layers is automatically filtered out. 


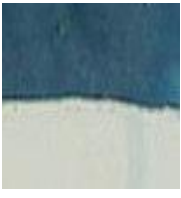

(a)

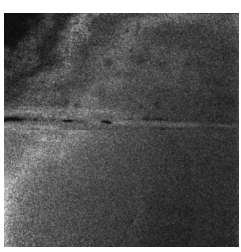

(b)

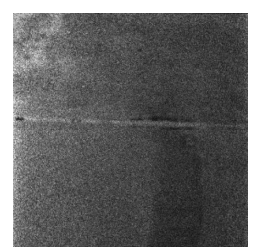

(c)

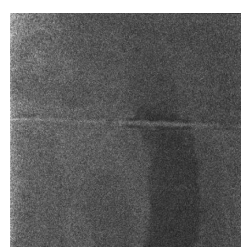

(d)

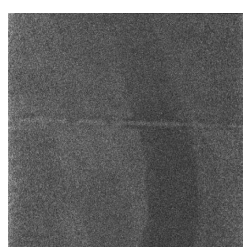

(e)

Fig. 4 a) Color image of a painted panel: the lower part is painted with an imprimatura on top of the underdrawing which is painted on a preparatory ground layer, the upper half has an additional paint layer above the imprimatura; b) average of the top four en-face images collected with System 1, i.e. 0-100 $\mu \mathrm{m}$; c) average of the next four en-face images (100-200 $\mu \mathrm{m})$; d) average of the next seven en-face images (200-375 $\mu \mathrm{m})$; e) average of the next seven images $(375-550 \mu \mathrm{m})$.
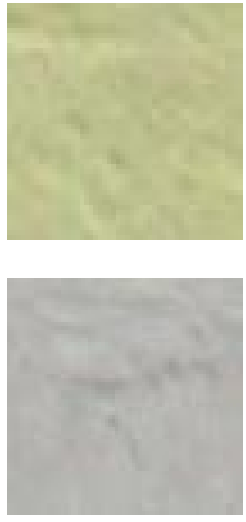

(a)
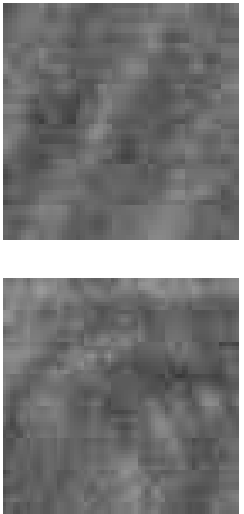

(b)
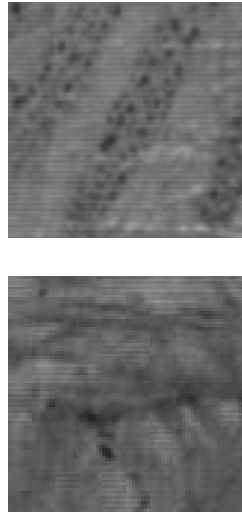

(c)
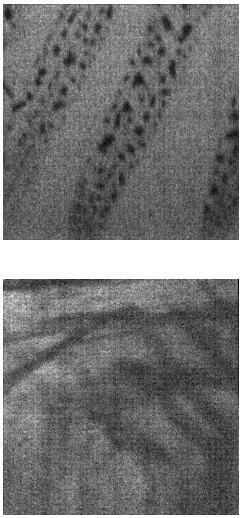

(d)

Fig. 5 a) Colour images of two painted patches over underdrawings: the top patch has two layers of lead-tin-yellow paint over underdrawings drawn with a quill pen using an ink of bone black in gum; the bottom patch has a mixture of lead white, azurite, bone black painted over a black chalk underdrawing; b) the corresponding near infrared Vidicon images; c) the corresponding near infrared images taken with a InGaAs camera; d) the corresponding $1300 \mathrm{~nm}$ OCT images taken with System 1 at the depth of the underdrawings.

\subsection{Refractive index measurements}

The traditional method of measuring refractive index (RI) of paint and varnish layers of a painting requires taking tiny samples and observing under a microscope the changes in their transparency when immersed in a series of liquids with calibrated $\mathrm{RI}^{17}$. When the RI of the sample matches that of the liquid, the sample 'disappears'. This method clearly requires the sample to be transparent under visible light and the measured refractive index is the average over the visible spectrum. For example, a number of tiny samples from a glass plate with mastic varnish applied in 1952 were used to measure the RI using this method and after 5 trials the average RI in the visible was measured to be $1.537 \pm 0.003$.

OCT is capable of measuring RI non-invasively for a wider range of paint media since OCT operates in the near infrared and most paints are more transparent in the near infrared than in the visible. There are two ways of measuring the refractive index of a medium using OCT. The simplest method is to measure the ratio of the optical path difference (OPD) in the medium and the OPD in air where RI is given by $n=1_{\text {med }} / 1_{\text {air. }}$ This is demonstrated in Fig.6 for the 1952 mastic varnish on glass where the gap in the varnish layer allowed the OPD in air and in the medium to be measured at the same point. The refractive indices at the two edges are measured to be $1.529 \pm 0.012$ and $1.524 \pm 0.012$ at $1300 \mathrm{~nm}$ using System 1, which means the thickness of the varnish layer in air is $\sim 90 \mu \mathrm{m}$. The measurements are consistent with 
the results obtained using the immersion method. This simple optical path length method is restricted to edges and areas where the top layer has cracked. Another method based on combined focus and OPD tracking ${ }^{13}$ can be used to measure the refractive index anywhere on a painting. First the lens is focused on the top of the varnish sample with the reference arm adjusted to match the OPD, then the lens is focused on the bottom of the varnish layer again with the OPD of the reference arm adjusted. The difference in the two OPDs and the difference in the two focus positions determine the refractive index. In the limit of a small NA, the refractive index is given by $n=\sqrt{ }(1+\Delta \mathrm{l} / \Delta \mathrm{z})$, where $\Delta \mathrm{z}$ is the difference in focus position and $\Delta \mathrm{l}$ is the difference between the two OPDs. We measured with System 3 the same 1952 mastic varnish using this method at two random spots on the varnish and found the refractive indices to be $1.557 \pm 0.028$ and $1.542 \pm 0.024$ at $1300 \mathrm{~nm}$, which are consistent with the results obtained from the two other methods described above. The accuracy can be improved significantly by increasing the resolution of the micrometer stage which is currently adjusted and read-out manually.

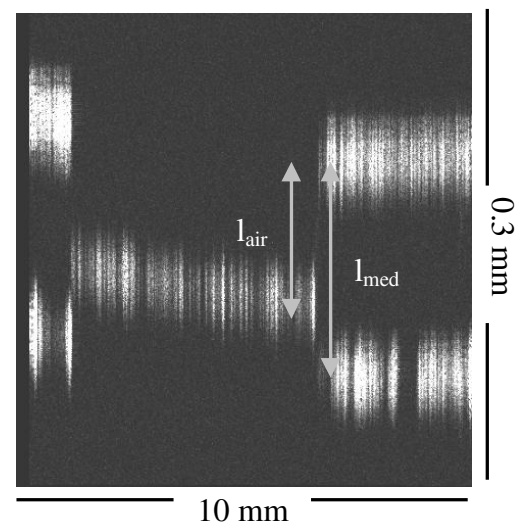

Fig. 6 A cross-section image of a mastic varnish on a glass plate (prepared and applied in 1952) obtained with System 1.

\subsection{Varnish drying process}

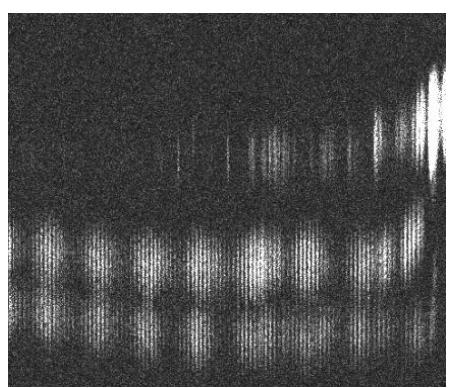

(a)

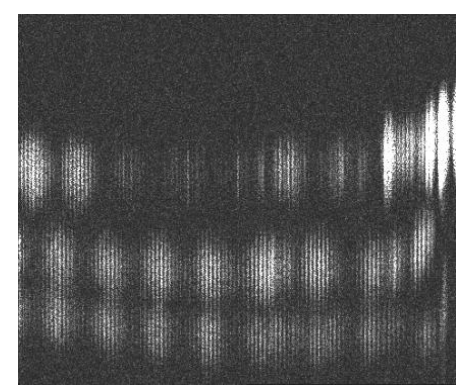

(b)

Fig. 7 Drying process for a Paraloid B72 varnish on a regular rough surface with peaks (and troughs) at $80 \mu \mathrm{m}$ intervals, top layer is the reflection from the top of the varnish, the middle layer is the top of the peaks the rough surface and the bottom layer is the troughs of the rough surface. A cross-section image taken with System 1 ten minutes after the application of the varnish is given in a), an image of the same position ten minutes later is given in $b$ ).

It was known in the $15^{\text {th }}$ century that application of varnish not only gives the painting a uniform gloss but also increases the colour saturation of the underlying paint layer ${ }^{18}$. It is well known that different types of varnish give different optical 
appearances to a painting, with natural resins such as mastic and dammar being the preferred types of varnish. It has been assumed that the optical difference is a result of the difference in RI of the different types of varnish, until recent work found that it was the ability of the varnish to level the surface of the paint rather than the refractive index differences that made one varnish preferable to another ${ }^{19}$.

OCT provides a convenient way of monitoring the wetting and drying process of paint and varnish layers. We have conducted preliminary studies of the drying process of varnish using an OCT. A set of regular rough surfaces were prepared where SU8 microscopic pillars of given size and separation were cured with ultraviolet light on a piece of glass $^{20}$. Different types and concentration of varnish were applied to the surfaces. Fig.7 shows the drying process for a Paraloid B72 varnish (volume concentration 37\%) applied on a surface with regular pillars ( 40 $\mu$ m in diameter and height) approximately $80 \mu \mathrm{m}$ apart. As we can see in Fig.7, the surface becomes less smooth and starts to follow the roughness of the substrate as it dries, confirming the suggestion made in Berns \& de la Rie ${ }^{19}$.

\section{CONCLUSIONS}

Optical Coherence Tomography, a low coherence 3-D scanning Michelon's interferometer, is a powerful non-invasive technique for probing into the depth of paintings, providing 3-D infrared image cubes that can show not only the structure of the paint and varnish layers but also reveal the underdrawings and their depth positions. Its advantage over normal cross-section examination under a microscope being that it would be possible to make a cross-sectional examination across the surface of the paintings rather than only at those discrete points selected for sampling and it is non-invasive. It gives the best dynamic range and resolution images of underdrawings compared to any currently employed technique, since interferometers take advantage of the coherence properties of light. En-face OCT is particularly suited to the examination of paintings. We have extended our preliminary studies ${ }^{7}$ to the examination of underdrawings and quantitative measurements of the refractive index and thickness of varnish layers, as well as the study of the drying process of varnish.

\section{ACKNOLEDGEMENT}

H.L. would like to thanks Carl Evans, Neil Shirtcliff and Mike Newton of Nottingham Trent University for useful discussion and help with making the SU8 rough surfaces; Joyce Townsend of the Tate for help with measuring varnish RI using the immersion method under a microscope; other members of the Scientific and Conservation Departments of the National Gallery for supplying samples, test paintings and images, in particular Catherine Higgitt for preparing the varnish; the Applied Optics Group of the University of Kent for access to their instruments, assistance and hospitality during her visits.

\section{REFERENCES}

1 N. Khandekar, "Preparation of cross-sections from easel paintings", Reviews in Conservation, 4, 52, 2003.

2 D. Bertani, M. Cetica, G. Molesini, "Holographic tests on the Ghiberti panel, The Life of Joseph", Studies in Conservation, 27, 61, 1982.

3 D. Paoletti and G. Schirripa Spagnolo, "Automated digital speckle pattern interferometry contouring in artwork surface inspection”, Optical Engineering, 32(6), 1348, 1993.

4 K. Polikreti, A. Othonos, C. Christofides, "Optical characterization of varnish films by spectroscopic ellipsometry for application in artwork conservation”, Applied Spectroscopy, 59(1), 69, 2005.

5 R. M. A. Azzam, N. M. Bashara, Ellipsometry and Polarized light, Amsterdam: North Holland, 1977.

6 R. Rajadhyaksha, R. Anderson and R. Webb, "Video-rate confocal scanning laser microscope for imaging human tissues in vivo," Applied Optics, 38, 2105, 1999.

7 H. Liang, M. Gomez Cid, R. Cucu, G. Dobre, D. Jackson, C. Pannell, J. Pedro, D. Saunders, A. Podoleanu, "Application of OCT to examination of easel paintings", Second European Workshop on Optical Fibre Sensors, Proc. SPIE, 5502, $378,2004$.

8 M.-L. Yang, C.-W. Lu, I.-J. Hsu, C. C. Yang, "The use of optical coherence tomography for monitoring the subsurface morphologies of archaic jades", Archaeometry, 46(2), 171, 2004.

9 P. Targowski, B. Rouba, M. Wojtkowski, and A. Kowalczyk, "The application of optical coherence tomography to nondestructive examination of museum objects", Studies in Conservation, 49(2), 107, 2004.

10 D. Huang, E. A. Swanson, C. P. Lin, J. S. Schuman, W. G. Stinson, W. Chang, M. R. Hee, T. Flotte, K. Gregory, C. A. Puliafito, J. G. Fujimoto, "Optical coherence tomography”, Science, 254, 1178, 1991. 
11 W. Drexler, "Ultrahigh-resolution optical coherence tomography", Journal of Biomedical Optics, 9, 47, 2004.

12 A. Gh. Podoleanu, M. Seeger, G. M. Dobre, D. J. Webb, D. A. Jackson and F. Fitzke "Transversal and longitudinal images from the retina of the living eye using low coherence reflectometry," Journal of Biomedical Optics, 3, 12, 1998.

13 G. J. Tearney, M. E. Brezinski, J. F. Southern, B. E. Bouma, M. R. Hee and J. G. Fujimoto, "Determination of the refractive index of highly scattering human tissue by optical coherence tomography", Optics Letters, 20(21), 2258, 1995.

14 A. Gh. Podoleanu, J. A. Rogers, D. A. Jackson and S. Dunne, "Three dimensional OCT images from retina and skin", Optics Express, 7(9), 292, 2000. http://www.opticsexpress.org/framestocv7n9.htm.

15 B. R. Masters, "Three-dimensional confocal microscopy of the human optic nerve in vivo," Opt. Express, 3, 356-359 (1998), http://epubs.osa.org/oearchive/source/6295.htm.

16 A. Gh. Podoleanu, G. M. Dobre, D. J. Webb, D. A. Jackson, "Coherence imaging by use of a Newton rings sampling function", Optics Letters, 21(21), 1789, 1996.

17 J. H. Townsend, "The Refractive Index of 19th-Century Paint Media: A Preliminary Study", ICOM Committee for Conservation, Working Group 16, Vol. II, 586, 1993.

18 C. Cennini, "Il Libro Dell'Arte" - The Craftsman's Handbook translated by D. V. Thompson Jr., Dover Publications Inc., New York, p99, 1954.

19 R. S. Berns and E. R. de la Rie, "Exploring the optical properties of picture varnishes using imaging techniques", Studies in Conservation, 48(2), 73-83, 2003.

20 N. J. Shirtcliffe, S. Aqil, C. Evans, G. McHale, M. I. Newton, C. C. Perry and P. Roach, "The use of high aspect ratio photoresist (SU8) for super-hydrophobic pattern prototyping”, Journal of Micromechanics \& Microengineering, 14, $1384,2004$. 